\section{High-Efficiency Cloning System for Versatile Adaptation of DNA Fragments}

BioTechniques 33:738-742 (October 2002)

Recombinant DNA vectors are fundamental tools in molecular biology and genetic engineering. One of the problems frequently encountered in vector construction is the lack of compatible restriction sites between the vectors and the DNA inserts. The conventional approach to solve this problem is to make the DNA fragments blunt-ended $(3,4,12)$ or to add linkers or adaptors $(10,11,14)$ before ligation. However, the efficiency of using blunt-end ligation and linkers/adaptors is low. The addition of linkers or adaptors can be expensive because a new linker/ adaptor has to be made for insertion at each new restriction site. Moreover, they are not commercially available for all sites. As PCR is one of the most important techniques in the field of recombinant DNA, the introduction of new restriction sites to DNA fragments by PCR has become an alternative method $(1,6,13)$. However, PCR-directed mutagenesis requires prior knowledge of the flanking sequence of the targeted fragments to design the primers and to synthesize the oligonucleotide. It also takes time to characterize the optimal conditions before large-scale PCR amplification. The main disadvantages of PCR-generated mutagenesis are as follows. (i) There exists a size limitation on the fragments that can be amplified by PCR. Introduction of new restriction sites by PCR may not be applicable to large DNA fragments. The size limitation of DNA fragments obtained by high-fidelity PCR fragments is less than $5 \mathrm{~kb}(2)$. Although currently longer and more accurate DNA amplification can be achieved by using high-fidelity DNA polymerases with $3^{\prime} \rightarrow 5^{\prime}$ proofreading exonuclease activity $(2,8)$, amplifying fragments in excess of $20 \mathrm{~kb}$ in length is still problematic, especially from complex templates such as genomic DNA $(1,2)$. (ii) Sequence analysis is required for each PCR-amplified fragment (1). Even with high-fidelity DNA polymerases, the en- tire amplified fragment has to be sequenced to insure there are no DNA polymerase-derived mutations. Furthermore, several rounds of sequence analysis are required for any fragment larger than 800 bp because the reliable sequence obtained from each sequencing reaction is $500-800$ bp (1). New primers have to be synthesized for each round of sequencing analysis. (iii) Primer design and synthesis, as well as the entire PCR process, have to be repeated if the same DNA fragment needs to be inserted into different sites.

To overcome these difficulties, our laboratory has developed a novel cloning system, pLinus. By using simple, inexpensive, and efficient basic cloning techniques, pLinus vectors can adapt DNA fragments from various sources in a single step to 32 unique restriction sites simultaneously, thus making the repeat procedure unnecessary. Here we discuss the features and advantages of this user-friendly pLinus cloning system.

The pLinus system consists of two sets of plasmids, pLinus16 (Figure 1, A and $\mathrm{B}$ ) and pLinus 17 (Figure 2, A and B). Both pLinus 16 and pLinus 17 consist of a pair of plasmids derived from pLitmus 28/29 and 38/39 (New England Biolabs, Beverly, MA, USA) respectively. They provide a total of 29 unique restriction sites with cohesive ends and three restriction sites (SnaBI, EcoRV, and StuI) for blunt-end ligations. Each pLinus plasmid contains two inverted repeated copies of the multiple cloning sites flanking a central stuffer fragment derived from either $\lambda$ DNA or enhanced GFP (EGFP) sequence. The 780-bp stuffer piece in pLinus 16-Apa was an EGFP gene fragment, cut with restriction endonuclease $A p a \mathrm{I}$ from the pcDNA3.1-EGFP plasmid (data not shown). The pcDNA3.1-EGFP was constructed by insertion of a 758-bp KpnI/ NotI EGFP fragment from pEGFP-1 (BD Biosciences Clontech, Palo Alto, CA, USA) into the KpnI/NotI sites of pcDNA3.1 (Invitrogen, Carlsbad, CA, USA). The two inverted repeated copies of the multiple cloning site in each pLinus vector allow versatile insertion of DNA fragments and provide a cassette such that DNA pieces can be cut out and cloned into other vectors, thus making the subsequent handling of DNA pieces 
much easier and faster. The stuffer piece helps to stabilize the plasmid structure by preventing the potential formation of secondary DNA structures between the two inverted repeated sequences. It also two multiple cloning sites for double digestions to work efficiently and allows provides sufficient space between the

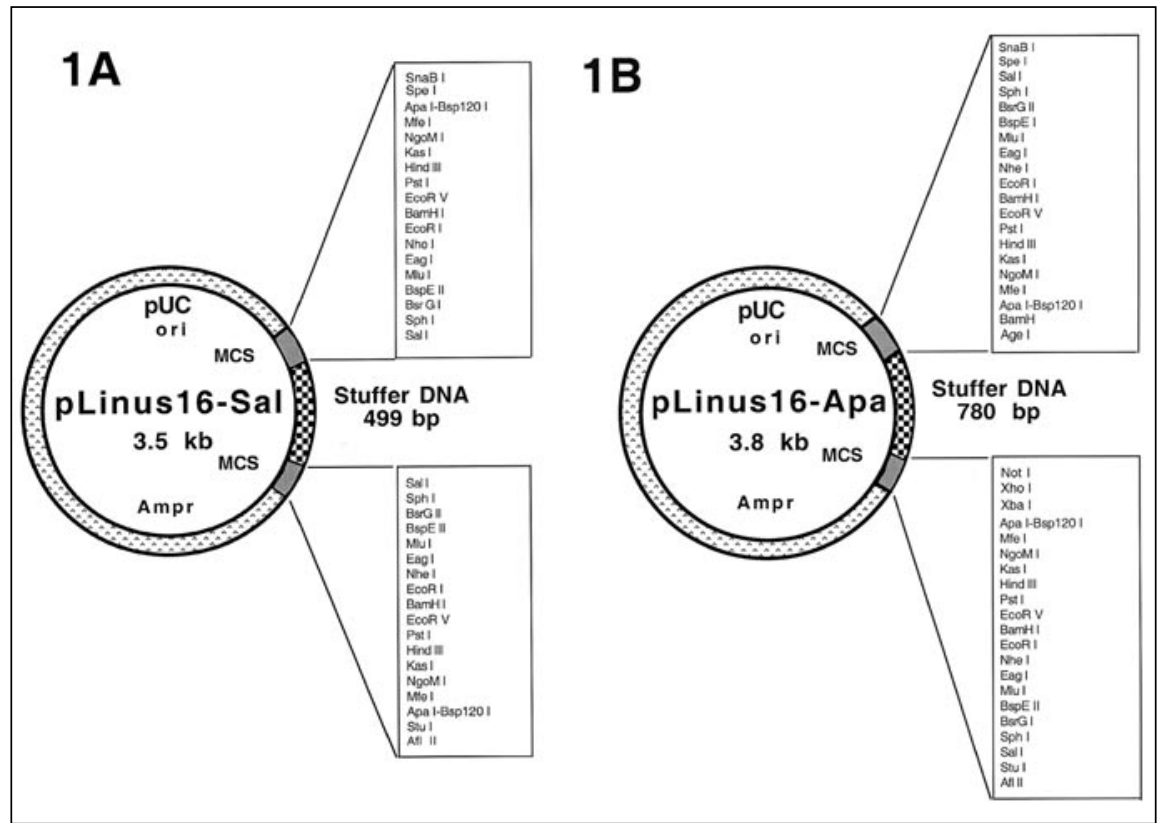

Figure 1. Schematics of pLinus16 vectors. (A) pLinus16-Sal was derived by ligating the 2250-bp AlwNI-SalI fragment of pLitmus 39 with the 685-bp AlwNI-SalI fragment of pLitmus 38. The stuffer DNA is a 499-bp SalI fragment from $\lambda$ DNA. (B) pLinus16-Apa was derived by ligating the 2250-bp $A l w$ NI-ApaI fragment of pLitmus 38 with the 685-bp AlwNI-ApaI fragment of pLitmus 39 . The stuffer DNA is a 780-bp ApaI fragment consisting of EGFP sequence.

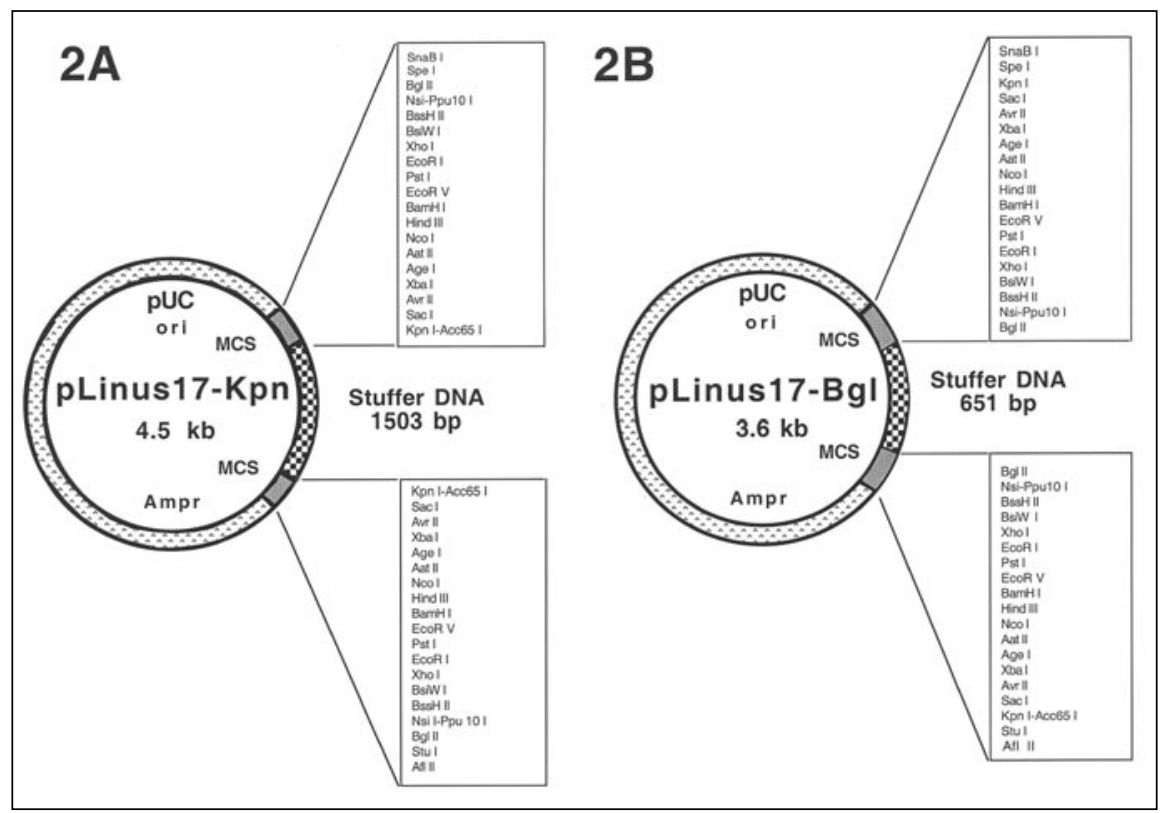

Figure 2. Schematics of pLinus17 vectors. (A) pLinus17-Kpn was derived by ligating the 2250-bp $A l w$ NI-KpnI fragment of Litmus29 with the 695-bp AlwNI-KpnI fragment of Litmus 28. The stuffer DNA is a 1503-bp KpnI fragment from $\lambda$ DNA. (B) pLinus17-Bgl was derived by ligating the 2250-bp $A l w$ NI-BglII fragment of Litmus 28 with the 695-bp AlwNI-BglII fragment of Litmus 29 . The stuffer DNA is a 651 -bp $B g l I I$ fragment from $\lambda$ DNA. verification that the digestion is completed by gel electrophoresis.

DNA fragments with overhangs compatible to only one of the 32 restriction sites in the multiple cloning site of the pLinus systems can be easily adapted for any of the other remaining 31 sites by first cloning the inserts into one of the pLinus vectors and then releasing the inserts from any pair of flanking restriction sites.

DNA inserts with two different overhangs compatible to restriction sites in the multiple cloning sites of the pLinus plasmids can also be adapted to new restriction sites. This can be accomplished by a complete digestion of the pLinus vector at the inner double-flanking sites followed by a partial digestion of the outer sites. The DNA fragment then ligates to the partially digested pLinus vector. It should be noted that once the DNA fragment is inserted into the pLinus, it has simultaneously been adapted for most of the commonly used restriction sites such as EcoRI and HindIII, resulting in significant cost-savings. It can further be excised and cloned into other expression vectors with any of these restriction sites without having to repeat the adaptation process, thus saving a considerable amount of time. We have successfully used AgeI/XbaI sites of pLinus17-Kpn to adapt the 469-bp SgrAI-NheI mouse GM-CSF gene from pORF-mGM-CSF (Invivogen, San Diego, CA, USA) for cloning into the XhoI site of an expression vector pCMV-MCS-neo constructed by our laboratory (data not shown).

DNA fragments with overhangs incompatible to any of the restriction sites within the pLinus polylinkers can first be rendered blunt-ended by either exonuclease or DNA polymerase treatment and then ligated into the blunt-ended site (EcoRV) present in the multiple cloning site of each pLinus vector. Once inserted, the DNA fragment can then be excised at the appropriate flanking sites to yield a fragment with cohesive ends of choice. This approach works more efficiently than direct blunt-end ligation between the DNA inserts and vectors for two reasons: $(i)$ the pLinus plasmids are much smaller than most expression vectors and (ii) once the DNA fragment has been inserted into pLinus, it can be cut back out by many different restriction 
enzymes to generate cohesive ends for subcloning.

Unmodified PCR products can also be directly adapted for multiple sites using the pLinus cloning systems. In most cases, the naturally occurring compatible restriction sites in PCR fragments are not present. Cloning of the PCR products mainly relies on the TA cloning method $(7,17)$, which exploits the fact that most unmodified PCR products have an A overhang at the $3^{\prime}$-ends. Such fragments can first be adapted to the commercially available TA cloning vectors before subcloning into the desired vectors, or they can be directly ligated to the modified vectors bearing single $3^{\prime}$-T overhangs at both ends $(7,9,15-17)$. However, the commercial TA cloning vectors are costly, and not all vectors can be modified into $\mathrm{T}$ vectors. Each of the four pLinus plasmids can be converted into a $\mathrm{T}$ vector by digestion with the EcoRV enzyme, followed by incubation with Taq DNA polymerase and dTTP. The advantage of this approach is that the PCR products would be simultaneously adapted for most of the commonly used restriction sites. Such PCR products can then be easily subcloned into other vectors at many different sites.
The potential for adapting DNA fragments of varying size is another significant advantage of pLinus vectors. One of the problems in introducing restriction sites by PCR is the size limitation for the amplified DNA fragments. Generally, even with high-fidelity DNA polymerases, it is impractical to introduce restriction sites to DNA fragments larger than $20 \mathrm{~kb}$ by PCR. The size limitation of pLinus vector for adaptation of large DNA inserts has not been investigated, but the great capacity of plasmid vectors for carrying DNA fragments as large as up to $74 \mathrm{~kb}$ has been reported (5). pLinus vectors have been used to adapt DNA fragments as small as $100 \mathrm{bp}$ (data not shown).

In summary, with its flexibility in the choice of 32 unique sites for the most commonly used restriction enzymes, the pLinus cloning system can provide highly efficient and versatile adaptation for routine cloning and manipulation of DNA fragments from various sources. Because it utilizes classical in vitro ligation/restriction techniques, saving both time and resources, it should be particularly useful for those who do not have easy access to all of the sophisticated modern cloning technologies.

\section{REFERENCES}

1.Ausubel, F.M., L.M. Albright, and J. Ju. 1999. Current Protocols in Molecular Biology. John Wiley \& Sons, New York.

2.Barnes, W.M. 1994. PCR amplification of up to $35-\mathrm{kb}$ DNA with high fidelity and high yield from $\lambda$ bacteriophage templates. Proc. Natl. Acad. Sci. USA 91:2216-2220.

3.Bhat, G.J., M.J. Lodes, P.J. Myler, and K.D. Stuart. 1991. A simple method for cloning blunt ended DNA fragments. Nucleic Acids Res. 19:398.

4.Damak, S. and D.W. Bullock. 1993. A simple two-set method for efficient blunt-end ligation. BioTechniques 15:448-452.

5.Darai, G. and H. Koch. 1985. Amplification and stability of recombinant plasmid molecules with a very large insert of foreign genetic material. Dev. Biol. Stand. 59:23-29.

6.Ito, W., H. Ishiguro, and Y. Kurosawa. 1991. A general method for introducing a series of mutations into cloned DNA using the polymerase chain reaction. Gene 102:67-70.

7.Horton, R.M., R. Raju, and B.M. ContiFine. 1997. A T-linker strategy for modification and directional cloning of PCR products. Methods Mol. Biol. 67:101-110.

8.Lundberg, K.S., D.D. Shoemaker, M.W. Adams, J.M. Short, J.A. Sorge, and E.J. Mathur. 1991. High-fidelity amplification using a thermostable DNA polymerase isolated from Pyrococcus furiosus. Gene 108:1-6.

9.Marchuk, D., M. Drumm, A. Saulino, and F.S. Collins. 1991. Construction of T-vectors, a rapid and general system for direct cloning of unmodified PCR products. Nucleic Acids Res. 19:154.

10.Perlman, D. and H.O. Halvorson. 1986. The MURTFI linker for multiple reading frame of a sense or nonsense codon into DNA. Nucleic Acids Res. 14:2139-2155.

11.Rothstein, R.J., L.F. Lau, C.P. Bahl, S.A. Narang, and R. Wu. 1979. Synthetic adaptors for cloning DNA. Methods Enzymol. 68:98-109.

12.Sambrook, J., E.F. Fritsch, and T. Maniatis. 1989. Molecular Cloning: A Laboratory Manual. CSH Laboratory Press, Cold Spring Harbor, NY.

13.Shimada, A. 1996. PCR-based site-directed mutagenesis. Methods Mol. Biol. 57:157-165.

14.Supak-Koslovsky, J.M. and M.D. Thomas. 1992. Subcloning using simplified adaptor addition. BioTechniques 13:226-230.

15.Tsang, T.C., D.T. Harris, E.T. Akporiaye, S.F. Schluter, G.T. Bowden, and E.M. Hersh. 1996. Simple method for adaptation DNA fragments and PCR products to all of the commonly used restriction sites. BioTechniques 20:51-52.

16.Testori, A., I. Listowsky, and P. Sollitti. 1994. Direct cloning of unmodified PCR products by exploiting an engineered restriction site. Gene 143:151-152.

17.Zhou, M.Y. and C.E. Gomez-Sanchez. 2000. Universal TA cloning. Curr. Issues Mol. Biol. 2:1-7.

Thanks to Deborah A. Schafer and Kathryn E. Huey-Tubman for their assistance in developing the graphics for the manuscript. Address correspondence to Dr. Phoebe Luo, Gene Therapy Group, Dept. of Microbiology and Immunology, University of Arizona, Tucson AZ, 85721, USA. e-mail: phoebe_luo@hotmail.com

Received 15 November 2001; accepted 24 June 2002.

P. Luo, T.C. Tsang, C.

Takeuchi, J. Dekker, M.

Badowski, and D.T. Harris

University of Arizona

Tucson, AZ, USA

For reprints of this or any other article, contact Reprints@BioTechniques.com 